Research Article

\title{
Edge Weight-Based Entropy of Magnesium Iodide Graph
}

\author{
Maryam Salem Alatawi $\mathbb{D},{ }^{1}$ Ali Ahmad $\mathbb{D}^{2}$, Ali N. A. Koam $\mathbb{D},{ }^{3}$ Sadia Husain $\mathbb{D}^{2}$ \\ and Muhammad Azeem iD ${ }^{4}$ \\ ${ }^{1}$ Department of Mathematics, Faculty of Sciences, University of Tabuk, Tabuk 71491, Saudi Arabia \\ ${ }^{2}$ College of Computer Science \& Information Technology, Jazan University, Jazan, Saudi Arabia \\ ${ }^{3}$ Department of Mathematics, College of Science, Jazan University, New Campus, Jazan 2097, Saudi Arabia \\ ${ }^{4}$ Department of Mathematics, Riphah Institute of Computing and Applied Sciences, Riphah International University, \\ Lahore, Pakistan
}

Correspondence should be addressed to Ali Ahmad; ahmadsms@gmail.com

Received 15 September 2021; Accepted 27 October 2021; Published 15 November 2021

Academic Editor: Ji Gao

Copyright $\odot 2021$ Maryam Salem Alatawi et al. This is an open access article distributed under the Creative Commons Attribution License, which permits unrestricted use, distribution, and reproduction in any medium, provided the original work is properly cited.

\begin{abstract}
Among the inorganic compounds, there are many influential crystalline structures, and magnesium iodide is the most selective. In the making of medicine and its development, magnesium iodide is considered a multipurpose and rich compound. Chemical structures and networks can be studied by given tools of molecular graph theory. Given tools of molecular graph theory can be studied for chemical structures and networks, which are considered economical with simple methodology. Edge weight-based entropy is a recent advent tool of molecular graph theory to study chemical networks and structures. It provides the structural information of chemical networks or their related build-up graphs and highlights the molecular properties in the form of a polynomial function. In this work, we provide the edge weight-based entropy of magnesium iodide structure and compute different entropies, such as Zagreb and atom bond connectivity entropies.
\end{abstract}

\section{Introduction}

Magnesium iodide is a chemical compound and known for its chemical formula $\mathrm{Mgl}_{2}$. Magnesium iodide is an inorganic compound that is used for synthesis in various organic substances, as well as it has other commercial uses. The major availability measures of $\mathrm{Mgl}_{2}$ are having their high impurity and volumes as a submicron and nanopowder. Magnesium iodide is obtained by the combined chemical mixture of hydro-iodic acid and magnesium carbonate and also the major chemical compounds magnesium oxide and magnesium hydroxide can be found. In the major applications of magnesium iodide, it is a highly valuable asset in internal medicine. By a unique pattern of $C_{4}$-graph, the molecular graph of magnesium iodide can be constructed. Having each $C_{4}$-graph inside, multiple heptagons are connected to each other [1]. For the easy readability and better understanding of the molecular graph of magnesium iodide, we labeled the parameters as $p$ is the number of $C_{4}$ 's of upper sides in a row and $q$ denoted for the count of lower side $C_{4}$ in heptagons. For all values of $q \in \mathbb{Z}$ with $q \geq 1$, magnesium iodide graph is needed to maintain for even and odd values of $p$ separately with the relation of $p=2(q+1)$ and $p=2 q+1$, respectively.

"The entropy of a probability distribution known as a measure of the unpredictability of information content or a measure of the uncertainty of a system." This quotation was the foundation, described in [2], as a seminal theory for the idea of entropy. Due to this concept is strongly based on statistical methodology, it became well-known for chemical structures and their corresponding graphs. This parameter provides a piece of extensive information about graphs, structures, and chemical topologies. In 1955, the notion and its idea were used first time for graphs. In sociology, ecology, biology, chemistry, and in a variety of other technical fields, graph-based entropy or simply entropy has applications $[3,4]$. Taking into consideration distinct graph elements associated with probability distributions, two types of 
entropy measurements are determined which are intrinsic and extrinsic entropies. The idea named degree-powers is a mathematical application of applied graph theory towards network theory to investigate networks as information functionals $[5,6]$. The physical sound of a network associated with the idea of entropy came forward from the authors in [7].

The major concern of this study is to determine some edge weight-based entropies of magnesium iodide structure for both cases of $p$. The methodology of this study of edge weight-based entropy is defined in Definitions 1-6, with their other fundamentals.

Definition 1. The first and second Zagreb index is introduced in 1972 by $[8,9]$ as

$$
\begin{aligned}
& M_{1}(F)=\sum_{\mathfrak{u} \mathfrak{v} \in E(F)}\left(\lambda_{\mathfrak{u}}+\lambda_{\mathfrak{v}}\right), \\
& M_{2}(F)=\sum_{\mathfrak{u} \mathfrak{v} \in E(F)}\left(\lambda_{\mathfrak{u}} \times \lambda_{\mathfrak{b}}\right) .
\end{aligned}
$$

Definition 2. The researcher in [10] introduced the atom bond connectivity index as

$$
\operatorname{ABC}(F)=\sum_{\mathfrak{u} \mathfrak{v} \in E(F)} \sqrt{\frac{\lambda_{\mathfrak{u}}+\lambda_{\mathfrak{v}}-2}{\lambda_{\mathfrak{u}} \times \lambda_{\mathfrak{v}}}}
$$

Definition 3. The geometric arithmetic index of a graph is introduced by [11] as

$$
\mathrm{GA}(F)=\sum_{\mathfrak{u} \mathfrak{v} \in E(F)} \frac{2 \sqrt{\lambda_{\mathfrak{u}} \times \lambda_{\mathfrak{v}}}}{\lambda_{\mathfrak{u}}+\lambda_{\mathfrak{v}}}
$$

Definition 4. In 2014, entropy for an edge weighted graph $F$ is introduced in [12]:

$$
\Omega_{\psi}(F)=-\sum_{\mathfrak{u}^{\prime} \mathfrak{v}^{\prime} \in E(F)} \frac{\psi\left(\mathfrak{u}^{\prime} \mathfrak{v}^{\prime}\right)}{\sum_{\mathfrak{u} \mathfrak{b} \in E(F)} \psi(\mathfrak{u} \mathfrak{b})} \log \left[\frac{\psi\left(\mathfrak{u}^{\prime} \mathfrak{v}^{\prime}\right)}{\sum_{\mathfrak{u} \mathfrak{b} \in E(F)} \psi(\mathfrak{u} \mathfrak{b})}\right],
$$

where $\psi(\mathfrak{t} \mathfrak{b})$ is a weight for an edge $\mathfrak{t} \mathfrak{b}$.

By letting the edge of weight equal to the main part of the topological index, Manzoor et al. [13, 14] introduced the following entropies for an edge weighted-based graph. The following are some important formulas for this research work and all these are based on equation (5).

Definition 5. The first and second Zagreb entropies are defined as follows $[14,15]$ :

$$
\begin{aligned}
& \Omega_{M_{1}}(F)=-\frac{1}{M_{1}(F)} \log \left[\prod_{\mathfrak{u} \mathfrak{b} \in E(F)}\left[\lambda_{\mathfrak{u}}+\lambda_{\mathfrak{v}}\right]^{\left[\lambda_{\mathfrak{u}}+\lambda_{\mathfrak{v}}\right]}\right]+\log \left(M_{1}(F)\right), \\
& \Omega_{M_{2}}(F)=-\frac{1}{M_{2}(F)} \log \left[\prod_{\mathfrak{u} \mathfrak{v} \in E(F)}\left[\lambda_{\mathfrak{u} \lambda \mathfrak{v}}\right]^{\left[\lambda_{\mathfrak{u} \lambda \mathfrak{b}}\right]}\right]+\log \left(M_{2}(F)\right) .
\end{aligned}
$$

Definition 6. The atom bond connectivity and geometric arithmetic entropies are defined as follows [13]:

$$
\begin{aligned}
& \Omega_{\mathrm{ABC}}(F)=-\frac{1}{\operatorname{ABC}(F)} \log \left[\prod_{\mathfrak{u} \mathfrak{v} \in E(F)}\left[\sqrt{\frac{\lambda_{\mathfrak{u}}+\lambda_{\mathfrak{v}}-2}{\lambda_{\mathfrak{u} \lambda \mathfrak{v}}}}\right]^{\left[\sqrt{\lambda_{\mathfrak{u}}+\lambda_{\mathfrak{v}}-2 / \lambda_{\mathfrak{u} \mathfrak{j}}}\right]}\right]+\log (\operatorname{ABC}(F)), \\
& \Omega_{\mathrm{GA}}(F)=-\frac{1}{\mathrm{GA}(F)} \log \left[\prod_{\mathfrak{u} \mathfrak{v} \in E(F)}\left[\frac{2 \sqrt{\lambda_{\mathfrak{u} \lambda \mathfrak{v}}}}{\lambda_{\mathfrak{u}}+\lambda_{\mathfrak{v}}}\right]^{\left[2 \sqrt{\lambda_{\mathfrak{u} \mathfrak{j}}} / \lambda_{\mathfrak{u}}+\lambda \mathfrak{v}\right]}\right]+\log (\mathrm{GA}(F)) .
\end{aligned}
$$

The topic of discussion of this study is closely related to the numerical descriptors or topological indices, so read the fundamentals and basics; we refer to see the recent cluster [16-23]. In the recent decade, this concept has been studied intensively and numerous literatures are available. We will discuss only limited recent most articles on this concept and few are left for the interest of readers [24-28].

To investigate and gain the contents of a network, the entropy formulas as put forward by [2], along with this, it helps to know about the structural information of networks 
and chemical structures [29]. The concept of edge weightbased entropy of a graph developed the applications and exploration in biological systems. For example, by creating a graph of chemical or any biological system, it has been used to investigate live organisms in the systems. For the biological and chemical applications of this study, see [30,31]. In computer science, in structural chemistry, and in even biology, the entropy can be found by [32]. This entropy, which is also explored in this study document, can be found in $[29,33]$ in-network heterogeneity work. In more recent literature about edge weight-based entropy, one can find $[34,35]$.

The edge weight-based entropies of the first and second Zagreb index, atom bond connectivity index, and geometric arithmetic index are figured out for the magnesium iodide or $\mathrm{Mgl}_{2}$ structure, for both even and odd cases of parameter $p$. The topological index of the magnesium iodide or $\mathrm{Mgl}_{2}$ structure, for both even and odd cases of parameter $p$, are computed in [1]. We will use the results of theorems from
[1], which are summarized in Tables 1 and 2. Moreover, due to long expressions of theorems, we reduced the calculations up to four decimal digits.

\section{Results on the Edge Weight-Based Entropy of Magnesium Iodide}

Given in this section are some important results of this research work. The idea is totally dependent on the structural values of $\mathrm{Mgl}_{2}$ or magnesium iodide graph, which is defined in Table 3 (for $p=$ odd and Table 4 (for $p=$ even, and the structure is shown in Figure 1.

Case 1. For the odd values of $p$ with given $q \geq 1$, let $p=$ $2 q+1$ and $q \in \mathbb{Z}$.

Theorem 1. Let $\Omega_{M_{1}}$ be the edge weight-based first Zagreb entropy for the $M G_{p, q}$ magnesium graph, with $p=2 q+1$, $q \geq 1$, then $\Omega_{M_{1}}\left(M G_{p, q}\right)$ is

$$
\Omega_{M_{1}}\left(\mathrm{MG}_{p, q}\right)=-\frac{1}{284 q+172} \log \left[2304 \cdot 4^{4} \cdot(25)^{5} \cdot(36)^{6} \cdot(343)^{7} \cdot(64)^{8} \cdot 9^{9} \times q(q+4)(q+5)(27 q-13)\right] .
$$

Proof. The edge partition of $\mathrm{MG}_{p, q}$ magnesium graph for the parameters $p=2 q+1$ and $q \geq 1$, given in Table 3, which are used to determine the topological indices of $M_{p, q}$, is summarized in Table 1 . Using the value of first Zagreb topological index from Table 1, along with the edge types from Table 3, in the formula defined in equation (6), after simplification, the entropy of first Zagreb resulted in

$$
\Omega_{M_{1}}(F)=-\frac{1}{284 q+172} \log \left[2304 \cdot 4^{4} \cdot(25)^{5} \cdot(36)^{6} \cdot(343)^{7} \cdot(64)^{8} \cdot 9^{9} \times q(q+4)(q+5)(27 q-13)\right] .
$$

Theorem 2. Let $\Omega_{M_{2}}$ be the edge weight-based second Zagreb entropy for the $M G_{p, q}$ magnesium graph, with $p=2 q+1$, $q \geq 1$, then $\Omega_{M_{2}}\left(M G_{p, q}\right)$ is

$$
\Omega_{M_{2}}\left(\mathrm{MG}_{p, q}\right)=-\frac{1}{543 q+190} \log \left[2304 \cdot 3^{3} \cdot 4^{4} \cdot(36)^{6} \cdot 8^{8} \cdot 9^{9} \cdot(10)^{10} \cdot(144)^{12} \cdot(15)^{15} \cdot(18)^{18} \times q(q+4)(q+5)(27 q-13)\right]
$$

Proof. Using the value of second Zagreb topological index from Table 1, along with the edge types from Table 3, in the formula defined in equation (7), after simplification, the entropy of second Zagreb resulted in

$$
\Omega_{M_{2}}\left(\mathrm{MG}_{p, q}\right)=-\frac{1}{543 q+190} \log \left[2304 \cdot 3^{3} \cdot 4^{4} \cdot(36)^{6} \cdot 8^{8} \cdot 9^{9} \cdot(10)^{10} \cdot(144)^{12} \cdot(15)^{15} \cdot(18)^{18} \times q(q+4)(q+5)(27 q-13)\right]
$$




\begin{tabular}{lc} 
& TABLE 1: Topological indices of $\mathrm{MG}_{p, q}$. \\
\hline$I$ & $I\left(\mathrm{MG}_{p, q}\right)$ \\
$M_{1}$ & $284 q+172$ \\
$M_{2}$ & $543 q+190$ \\
$\mathrm{ABC}$ & $21.1645 q+22.8602$ \\
$\mathrm{GA}$ & $30.8878 q+23.5189$ \\
For & $p=2 q+1, q \geq 1$. \\
\hline
\end{tabular}

\begin{tabular}{lc} 
& TABLE 2: Topological indices of $\mathrm{MG}_{p, q}$. \\
\hline$I$ & $I\left(\mathrm{MG}_{p, q}\right)$ \\
$M_{1}$ & $284 q+258$ \\
$M_{2}$ & $543 q+375$ \\
$\mathrm{ABC}$ & $21.1645 q+22.8602$ \\
$\mathrm{GA}$ & $30.8878 q+23.5189$ \\
For & $p=2(q+1), q \geq 1$. \\
\hline
\end{tabular}

TABLE 3: Edge partition of $\mathrm{MG}_{p, q}$ for $p=2 q+1, q \geq 1$.

\begin{tabular}{lcc}
\hline$\left(\lambda_{\mathfrak{u}}, \lambda_{\mathfrak{b}}\right)$ & Frequency & Set of edges \\
$(1,3)$ & 1 & $E_{1}$ \\
$(1,4)$ & 1 & $E_{2}$ \\
$(1,6)$ & $q+5$ & $E_{3}$ \\
$(2,3)$ & 2 & $E_{4}$ \\
$(2,4)$ & 2 & $E_{5}$ \\
$(2,5)$ & 8 & $E_{6}$ \\
$(2,6)$ & $2 q+8$ & $E_{7}$ \\
$(3,3)$ & $3 q$ & $E_{8}$ \\
$(3,4)$ & 1 & $E_{9}$ \\
$(3,5)$ & 12 & $E_{10}$ \\
$(3,6)$ & $27 q-13$ & $E_{11}$ \\
\hline
\end{tabular}

\begin{tabular}{lcc}
\multicolumn{2}{c}{ TABLE 4: Edge partition of $\mathrm{MG}_{p, q}$ for $p=2(q+1), q \geq 1}$. \\
\hline$\left(\lambda_{\mathfrak{u}}, \lambda_{\mathfrak{v}}\right)$ & Frequency & Set of edges \\
$(1,3)$ & 1 & $E_{1}$ \\
$(1,5)$ & 1 & $E_{2}$ \\
$(1,6)$ & $q+5$ & $E_{3}$ \\
$(2,2)$ & 2 & $E_{4}$ \\
$(2,3)$ & 2 & $E_{5}$ \\
$(2,5)$ & 8 & $E_{6}$ \\
$(2,6)$ & $2 q+8$ & $E_{7}$ \\
$(3,3)$ & $3 q$ & $E_{8}$ \\
$(3,5)$ & 1 & $E_{9}$ \\
$(3,6)$ & 12 & $E_{10}$ \\
\hline
\end{tabular}

Theorem 3. Let $\Omega_{A B C}$ be the edge weight-based atom bond connectivity entropy for the $M G_{p, q}$ magnesium graph, with $p=2 q+1, q \geq 1$, then $\Omega_{A B C}\left(M G_{p, q}\right)$ is

$$
\begin{aligned}
\Omega_{\mathrm{ABC}}\left(\mathrm{MG}_{p, q}\right)= & -\frac{1}{21.1645 q+22.8602} \\
& \log [4778.1804 q(q+4)(q+5)(27 q-13)] .
\end{aligned}
$$

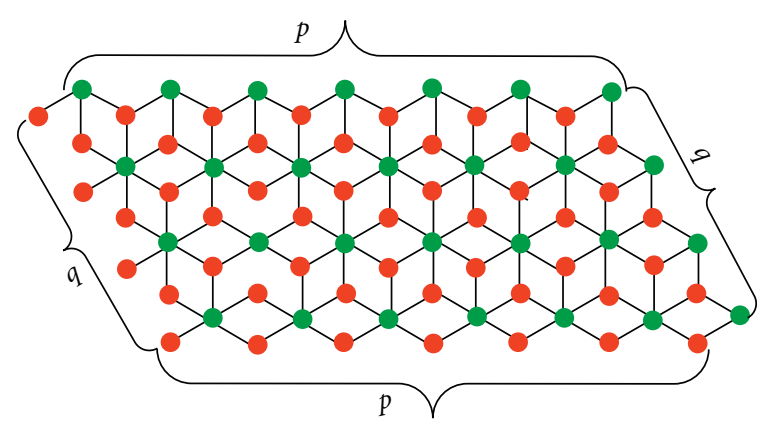

Figure 1: Magnesium iodide graph.

Proof. Using the value of atom bond connectivity topological index from Table 1, along with the edge types from Table 3, in the formula defined in equation (8), after simplification, the entropy of atom bond connectivity resulted in

$$
\begin{aligned}
\Omega_{\mathrm{ABC}}\left(\mathrm{MG}_{p, q}\right)= & -\frac{1}{21.1645 q+22.8602} \\
& \log [4778.1804 q(q+4)(q+5)(27 q-13)] .
\end{aligned}
$$

Theorem 4. Let $\Omega_{G A}$ be the edge weight-based geometric arithmetic entropy for the $M G_{p, q}$ magnesium graph, with $p=2 q+1, q \geq 1$, then $\Omega_{G A}\left(M G_{p, q}\right)$ is

$$
\begin{aligned}
\Omega_{\mathrm{GA}}\left(\mathrm{MG}_{p, q}\right)= & -\frac{1}{30.8878 q+23.5189} \\
& \log [949.8757 q(q+4)(q+5)(27 q-13)] .
\end{aligned}
$$

Proof. Using the value of geometric arithmetic topological index from Table 1, along with the edge types from Table 3, in the formula defined in equation (9), after simplification, the entropy of geometric arithmetic resulted in

$$
\begin{aligned}
\Omega_{\mathrm{GA}}\left(\mathrm{MG}_{p, q}\right)= & -\frac{1}{30.8878 q+23.5189} \\
& \log [949.8757 q(q+4)(q+5)(27 q-13)] .
\end{aligned}
$$

Case 2. For the even values of $p$ with given $q \geq 1$, let $p=$ $2(q+1)$ and $q \in \mathbb{Z}$.

Theorem 5. Let $\Omega_{M_{1}}$ be the edge weight-based first Zagreb entropy for the $M G_{p, q}$ magnesium graph, with $p=2(q+1)$, $q \geq 1$, then $\Omega_{M_{1}}\left(M G_{p, q}\right)$ is 


$$
\Omega_{M_{1}}\left(\mathrm{MG}_{p, q}\right)=-\frac{1}{284 q+258} \log \left[240 \cdot(16)^{4} \cdot 5^{5} \cdot 6^{6} \cdot(49)^{7} \cdot(64)^{8} \cdot(81)^{9} \times(q+4)(q+5)(3 q+1)(27 q+7)\right]
$$

Proof. The edge partition of $\mathrm{MG}_{p, q}$ magnesium graph for the parameters $p=2(q+1)$ and $q \geq 1$, given in Table 4 , which are used to determine the topological indices of $\mathrm{MG}_{p, q}$, is summarized in Table 2. Using the value of first
Zagreb topological index from Table 2, along with the edge types from Table 4, in the formula defined in equation (6), after simplification, the entropy of first Zagreb resulted in

$$
\Omega_{M_{1}}\left(\mathrm{MG}_{p, q}\right)=-\frac{1}{284 q+258} \log \left[240 \cdot(16)^{4} \cdot 5^{5} \cdot 6^{6} \cdot(49)^{7} \cdot(64)^{8} \cdot(81)^{9} \times(q+4)(q+5)(3 q+1)(27 q+7)\right]
$$

Theorem 6. Let $\Omega_{M_{2}}$ be the edge weight-based second Zagreb entropy for the $M G_{p, q}$ magnesium graph, with $p=2(q+1)$, $q \geq 1$, then $\Omega_{M_{2}}\left(M G_{p, q}\right)$ is

$$
\Omega_{M_{2}}\left(\mathrm{MG}_{p, q}\right)=-\frac{1}{543 q+375} \log \left[240 \cdot 3^{3} \cdot 4^{4} \cdot 5^{5} \cdot(36)^{6} \cdot 9^{9} \cdot(10)^{10} \cdot(12)^{12} \cdot(15)^{15} \cdot(18)^{18} \times(q+4)(q+5)(3 q+1)(27 q+7)\right]
$$

Proof. Using the value of second Zagreb topological index formula defined in equation (7), after simplification, the from Table 2, along with the edge types from Table 4, in the entropy of second Zagreb resulted in

$$
\Omega_{M_{2}}\left(\mathrm{MG}_{p, q}\right)=-\frac{1}{543 q+375} \log \left[240 \cdot 3^{3} \cdot 4^{4} \cdot 5^{5} \cdot(36)^{6} \cdot 9^{9} \cdot(10)^{10} \cdot(12)^{12} \cdot(15)^{15} \cdot(18)^{18} \times(q+4)(q+5)(3 q+1)(27 q+7)\right]
$$

Theorem 7. Let $\Omega_{A B C}$ be the edge weight-based atom bond connectivity entropy for the $M G_{p, q}$ magnesium graph, with $p=2(q+1), q \geq 1$, then $\Omega_{A B C}\left(M G_{p, q}\right)$ is

$$
\Omega_{\mathrm{ABC}}\left(\mathrm{MG}_{p, q}\right)=-\frac{1}{21.1645 q+22.8602} \log [2165.7809(q+4)(q+5)(3 q+1)(27 q+7)]
$$

Proof. Using the value of atom bond connectivity topological index from Table 2, along with the edge types from Table 4, in the formula defined in equation (8), after simplification, the entropy of atom bond connectivity resulted in

$$
\Omega_{\mathrm{ABC}}\left(\mathrm{MG}_{p, q}\right)=-\frac{1}{21.1645 q+22.8602} \log [2165.7809(q+4)(q+5)(3 q+1)(27 q+7)]
$$


Theorem 8. Let $\Omega_{G A}$ be the edge weight-based geometric arithmetic entropy for the $M G_{p, q}$ magnesium graph, with $p=2(q+1), q \geq 1$, then $\Omega_{G A}\left(M G_{p, q}\right)$ is

$$
\Omega_{\mathrm{GA}}\left(\mathrm{MG}_{p, q}\right)=-\frac{1}{30.8878 q+23.5189} \log [2895.9383(q+4)(q+5)(3 q+1)(27 q+7)]
$$

Proof. Using the value of geometric arithmetic topological index from Table 2, along with the edge types from Table 4, in the formula defined in equation (9), after simplification, the entropy of geometric arithmetic resulted in

$$
\Omega_{\mathrm{GA}}\left(\mathrm{MG}_{p, q}\right)=-\frac{1}{30.8878 q+23.5189} \log [2895.9383(q+4)(q+5)(3 q+1)(27 q+7)]
$$

\section{Conclusion}

The edge weight-based entropy of a network or structure provides structural information and detailed content in the form of mathematical equations. To add up some structural information and properties of magnesium iodide or $\mathrm{Mgl}_{2}$ structure, we determined the edge weight-based entropies of the first and second Zagreb index, atom bond connectivity index, and geometric arithmetic index. The results carried information for both even and odd cases of parameter $p$, of magnesium iodide, or $\mathrm{Mgl}_{2}$ structure.

\section{Data Availability}

There are no data associated with this article.

\section{Conflicts of Interest}

The authors declare that they have no conflicts of interest.

\section{References}

[1] F. Afzal, F. Asmat, and D. Afzal, "Molecular description for magnesium iodide," Mathematical Models in Engineering, vol. 5, no. 4, pp. 175-189, 2019.

[2] C. E. Shannon, "A mathematical theory of communication," Bell System Technical Journal, vol. 27, no. 3, pp. 379-423, 1948.

[3] M. Dehmer and M. Graber, "The discrimination power of molecular identification numbers revisited," MATCH Community of Mathematics and Computational Chemistry, vol. 69, pp. 785-794, 2013.

[4] R. E. Ulanowicz, "Quantitative methods for ecological network analysis," Computational Biology and Chemistry, vol. 28, no. 5-6, pp. 321-339, 2004.

[5] S. Cao, M. Dehmer, and Y. Shi, "Extremality of degree-based graph entropies," Information Sciences, vol. 278, pp. 22-33, 2014.

[6] S. Cao and M. Dehmer, "Degree-based entropies of networks revisited," Applied Mathematics and Computation, vol. 261, pp. 141-147, 2015.

[7] E. Estrada and N. Hatano, "Statistical-mechanical approach to subgraph centrality in complex networks," Chemical Physics Letters, vol. 439, no. 1-3, pp. 247-251, 2007.
[8] I. Gutman and N. Trinajstić, "Graph theory and molecular orbitals, total $\pi$-electron energy of alternant hydrocarbons," Chemical Physics Letters, vol. 17, no. 4, pp. 535-538, 1972.

[9] I. Gutman and K. C. Das, "The first zagreb index 30 years after," Match Community of Mathematics and Computational Chemistry, vol. 50, pp. 83-92, 2004.

[10] E. Estrada, L. Torres, L. Rodríguez, and I. Gutman, “An atombond connectivity index modeling the enthalpy of formation of alkanes," Indian Journal of Chemistry, vol. 37, pp. 849-855, 1998.

[11] D. Vukičević and B. Furtula, "Topological index based on the ratios of geometrical and arithmetical means of end-vertex degrees of edges," Journal of Mathematical Chemistry, vol. 46, pp. 1369-1376, 2009.

[12] Z. Chen, M. Dehmer, and Y. Shi, "A note on distance based graph entropies," Entropy, vol. 16, no. 10, pp. 5416-5427, 2014.

[13] S. Manzoor, M. K. Siddiqui, and S. Ahmad, "On entropy measures of molecular graphs using topological indices," Arabian Journal of Chemistry, vol. 13, no. 8, pp. 6285-6298, aug 2020.

[14] S. Manzoor, M. K. Siddiqui, and S. Ahmad, "On entropy measures of polycyclic hydroxychloroquine used for novel coronavirus (covid-19) treatment," Polycyclic Aromatic Compounds, 2020.

[15] X. Zuo, M. F. Nadeem, M. K. Siddiqui, and M. Azeem, "Edge weight based entropy of different topologies of carbon nanotubes," IEEE Access, vol. 9, pp. 102019-102029, 2021.

[16] M. F. Nadeem, M. Imran, H. M. Afzal Siddiqui, M. Azeem, A. Khalil, and Y. Ali, "Topological aspects of metal-organic structure with the help of underlying networks," Arabian Journal of Chemistry, vol. 14, no. 6, Article ID 103157, 2021.

[17] A. Shabbir, M. F. Nadeem, S. Mukhtar, and A. Raza, "On edge version of some degree-based topological indices of HAC5c7 [p,q] and VC5c7[p,q] nanotubes," Polycyclic Aromatic Compounds, pp. 1-17, 2020.

[18] M. F. Nadeem, M. Azeem, and H. M. A. Siddiqui, "Comparative study of zagreb indices for capped, semi-capped, and uncapped carbon nanotubes," Polycyclic Aromatic Compounds, pp. 1-18, 2021.

[19] M. F. Nadeem, M. Azeem, and I. Farman, "Comparative study of topological indices for capped and uncapped carbon nanotubes," Polycyclic Aromatic Compounds, pp. 1-18, 2021.

[20] M. F. Nadeem, S. Zafar, and Z. Zahid, "On topological properties of the line graphs of subdivision graphs of certain 
nanostructures," Applied Mathematics and Computation, vol. 273, pp. 125-130, 2016.

[21] A. Ahmad and S. C. López, "Distance-based topological polynomials associated with zero-divisor graphs," Mathematical Problems in Engineering, vol. 2021, Article ID 4959559, 8 pages, 2021.

[22] G. Hong, Z. Gu, M. Javaid, H. M. Awais, and M. K. Siddiqui, "Degree-based topological invariants of metal-organic networks," IEEE Access, vol. 8, pp. 68288-68300, 2020.

[23] S. Hayat, M. A. Malik, and M. Imran, "Computing topological indices of honeycomb derived networks," Romanian Journal of Information Science and Technology, vol. 18, pp. 144-165, 2015.

[24] A. Ali, K. C. Das, D. Dimitrov, and B. Furtula, "Atom-bond connectivity index of graphs: a review over extremal results and bounds," Discrete Mathematics Letters, vol. 5, no. 1, pp. 68-93, 2021.

[25] A. Ali, I. Gutman, E. Milovanovic, and I. Milovanovic, "Sum of powers of the degrees of graphs: extremal results and bounds," MATCH Communications in Mathematical and in Computer Chemistry, vol. 80, pp. 5-84, 2018.

[26] B. Borovicanin, K. C. Das, B. Furtula, and I. Gutman, "Bounds for zagreb indices," MATCH Communications in Mathematical and in Computer Chemistry, vol. 78, pp. 17-100, 2017.

[27] K. C. Das, I. Gutman, and B. Furtula, "Survey on geometric-arithmetic indicesof graphs," MATCH Communications in Mathematical and in Computer Chemistry, vol. 65, pp. 595-644, 2011.

[28] A. Portillaa, J. M. Rodriguezb, and J. M. Sigarreta, "Recent lower bounds for geometric-arithmetic index," Discrete Mathematics Letters, vol. 1, pp. 59-82, 2019.

[29] R. V. Sol and S. I. Valverde, "Information theory of complex networks: on evolution and architectural constraints," Complex Network Lectures Notes Physics, vol. 650, pp. 189207, 2004.

[30] N. Rashevsky, "Life, information theory, and topology," Bulletin of Mathematical Biophysics, vol. 17, no. 3, pp. 229235, 1955.

[31] E. Trucco, "A note on the information content of graphs," Bulletin of Mathematical Biophysics, vol. 18, no. 2, pp. 129135, 1956.

[32] M. Dehmer and A. Mowshowitz, "A history of graph entropy measures," Information Sciences, vol. 181, no. 1, pp. 57-78, 2011.

[33] Y. J. Tan and J. Wu, "Network structure entropy and its application to scale-free networks," System Engineering-Theory \& Practice, vol. 6, pp. 1-3, 2004.

[34] Y. C. Kwun, H. M. ur Rehman, M. Yousaf, W. Nazeer, and S. M. Kang, "The entropy of weighted graphs with atomic bond connectivity edge weights," Discrete Dynamics in Nature and Society, vol. 2018, Article ID 8407032, 10 pages, 2018.

[35] S. Sindhu, B. J. Gireesha, and G. Sowmya, "Entropy generation analysis of multi-walled carbon nanotube dispersed nanoliquid in the presence of heat source through a vertical microchannel," International Journal of Numerical Methods for Heat \& Fluid Flow, vol. 30, no. 12, pp. 5063-5085, 2020. 